\title{
Angioimmunoblastic T Cell Lymphoma Mimicking Postoperative Lymph Node Recurrence in Cholangiocarcinoma
}

\author{
Seungjung $\mathrm{Ha}^{1}$, Min Eui Hong ${ }^{2}$, Jae Keun Park ${ }^{1}$ \\ 'Division of Gastroenterology and Hepatology, Department of Internal Medicine, Kangnam Sacred Heart Hospital, Hallym University \\ College of Medicine, ${ }^{2}$ Department of Pathology, Kangnam Sacred Heart Hospital, Hallym University College of Medicine, Seoul, Korea
}

Received May 12, 2021, Revised May 24, 2021, Accepted May 24, 2021

Corresponding author: Jae Keun Park, E-mail: jaekpark@hallym.or.kr, https://orcid.org/0000-0002-3367-1770

\section{CASE SUMMARY}

An 81-year-old female presented with 2 weeks history of diffuse abdominal pain, general weakness and non-tender neck mass (Right $>$ Left). The initial vital sign in this visit was within normal range. She had a history of pyloruspreserving pancreaticoduodenectomy (PPPD) due to distal common bile duct (CBD) cancer six months ago in same hospital. On the surgical specimen, biopsy result reported the moderately differentiated adenocarcinoma with lymph node (LN) metastasis in 5 out of 12 LNs (pT3N2M0). Patient has been received the adjuvant radiotherapy $(4,860$ cGy/180 cGy/27 times) without chemotherapy considering her general condition (ECOG status 2-3) following a consultation with an oncologist.

The results of complete blood count were normal except mild thrombocytopenia (white blood count [WBC] 6,920/ $\mathrm{mm}^{3}$, hemoglobin [Hb] $12.2 \mathrm{~g} / \mathrm{dl}$, platelets [PLT] 131,000/
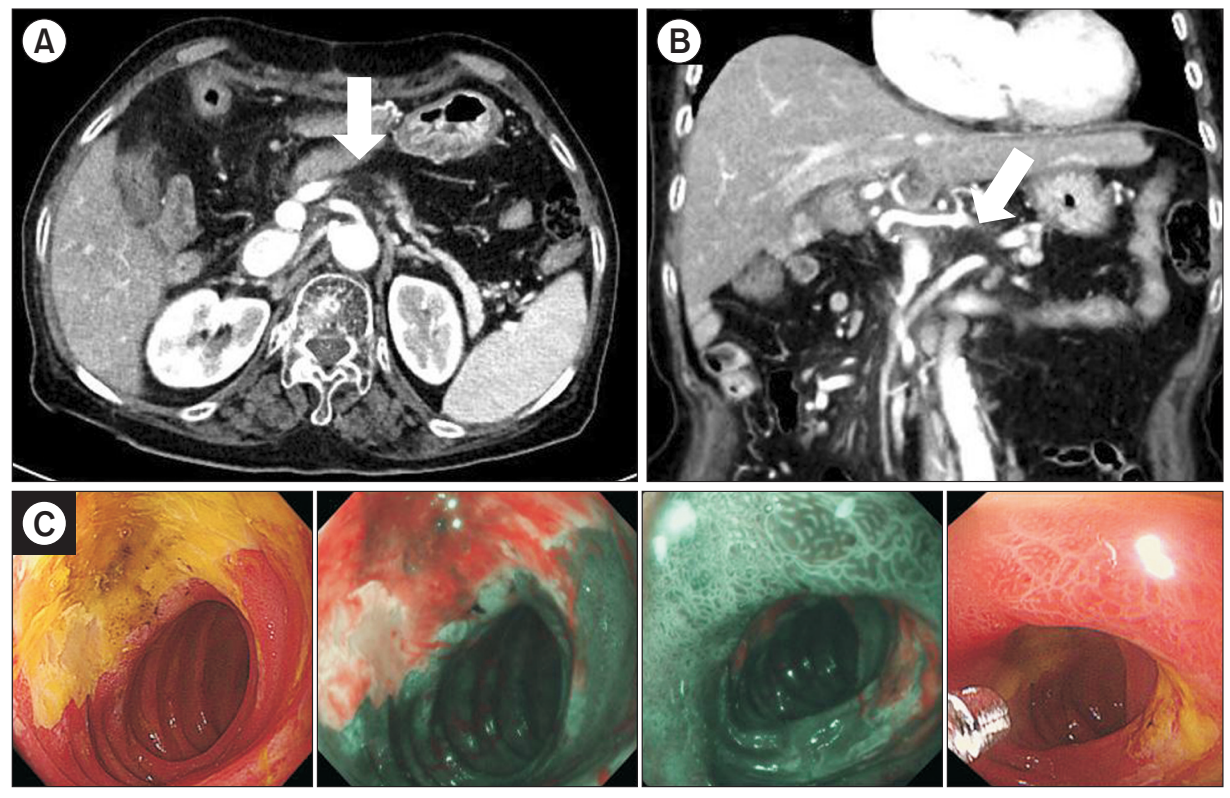

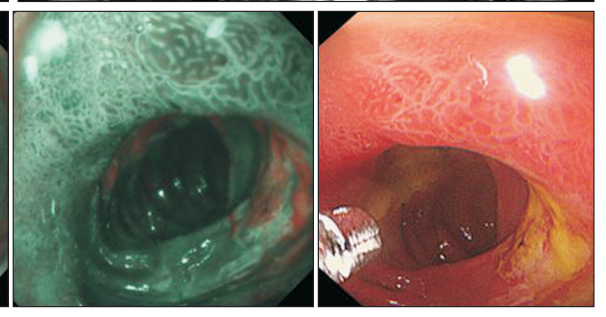

Fig. 1. (A) Cross section view of contrast enhanced computed tomography (CT) showing $15 \mathrm{~mm}$ enlargement of paraaortic lymph node (white arrow). (B) Coronal view of the contrast enhanced CT showing $15 \mathrm{~mm}$ enlargement of paraaortic lymph node (white arrow). (C) Endoscopy showing multiple ulcers with granulated tissue in the anastomosis site of pylorus-preserving pancreaticoduodenectomy (PPPD).

Copyright (C) Korean Society of Gastrointestinal Cancer Research. All riguts reserved.

(c) (1) (s) This is an Open Access article distributed under the terms of the Creative Commons Attribution Non-Commercial License (http://creativecommons.org/licenses/ by-nc/4.0). which permits unrestricted non-commercial use, distribution, and reproduction in any medium, provided the original work is properly cited. 
$\mathrm{mm}^{3}$ ). Serological test for liver function was normal (aspartate aminotransferase [AST] $29 \mathrm{U} / \mathrm{L}$, alanine aminotrans-
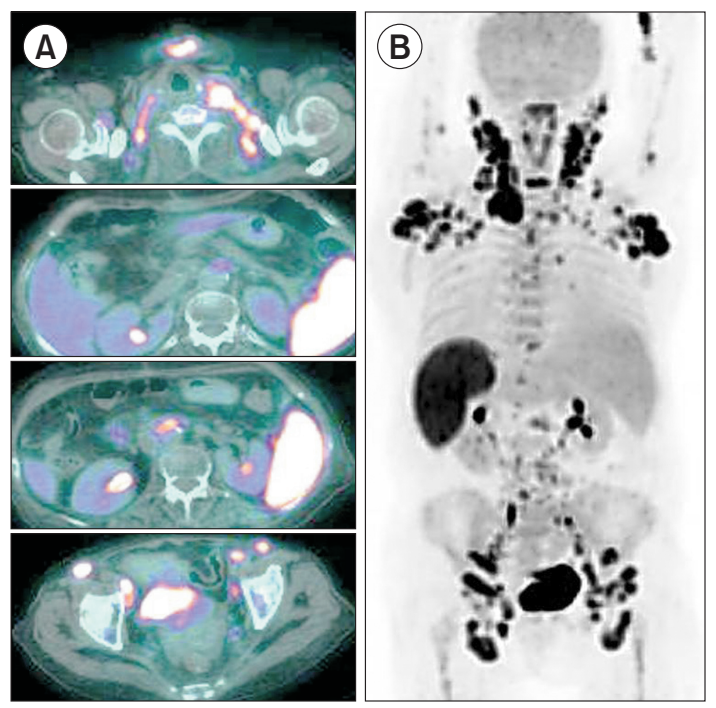

Fig. 2. Positron emission tomography/computed tomography (PET/CT) showing multiple lymphomatous involvement. (A) Cross section view. (B) Coronal view of PET/CT. ferase [ALT] $6 \mathrm{U} / \mathrm{L}$, alkaline phosphatase [ALP] $122 \mathrm{U} / \mathrm{L}$, gamma-glutamyl transpeptidase [GGT] $57 \mathrm{U} / \mathrm{L}$, total bilirubin $1.1 \mathrm{mg} / \mathrm{dl}$, direct bilirubin $0.4 \mathrm{mg} / \mathrm{dl}$ ) as were serum Carbohydrate Antigen (CA 19-9) and Carcinoembryonic Antigen (CEA) levels (CA 19-9 2.2 U/ml, CEA 0.6 ng/ml). Contrast enhanced computed tomography (CT) showed paraaortic LNs enlargement with splenomegaly (Fig. 1A, 1B), and esophagoduodenoscopy showed multiple ulcers with granulated tissue in the anastomosis site of PPPD (Fig. 1C). Endoscopic biopsies on anastomosis site were negative for malignancy. A positron emission tomography/computed tomography (PET/CT) study conducted for searching the local recurrence of CBD cancer showed fluorodeoxyglucose uptake in lymphomatous involvement of multiple lymph nodes including both cervical chains, both axillae, right 2 nd internal mammary, mediastinum, upper abdomen, retroperitoneum, both iliac chains, both inguinal areas, nasopharynx, tonsils, and spleen (Fig. 2A, 2B).

Since there was $2 \mathrm{~cm}$ sized right neck palpable mass, we
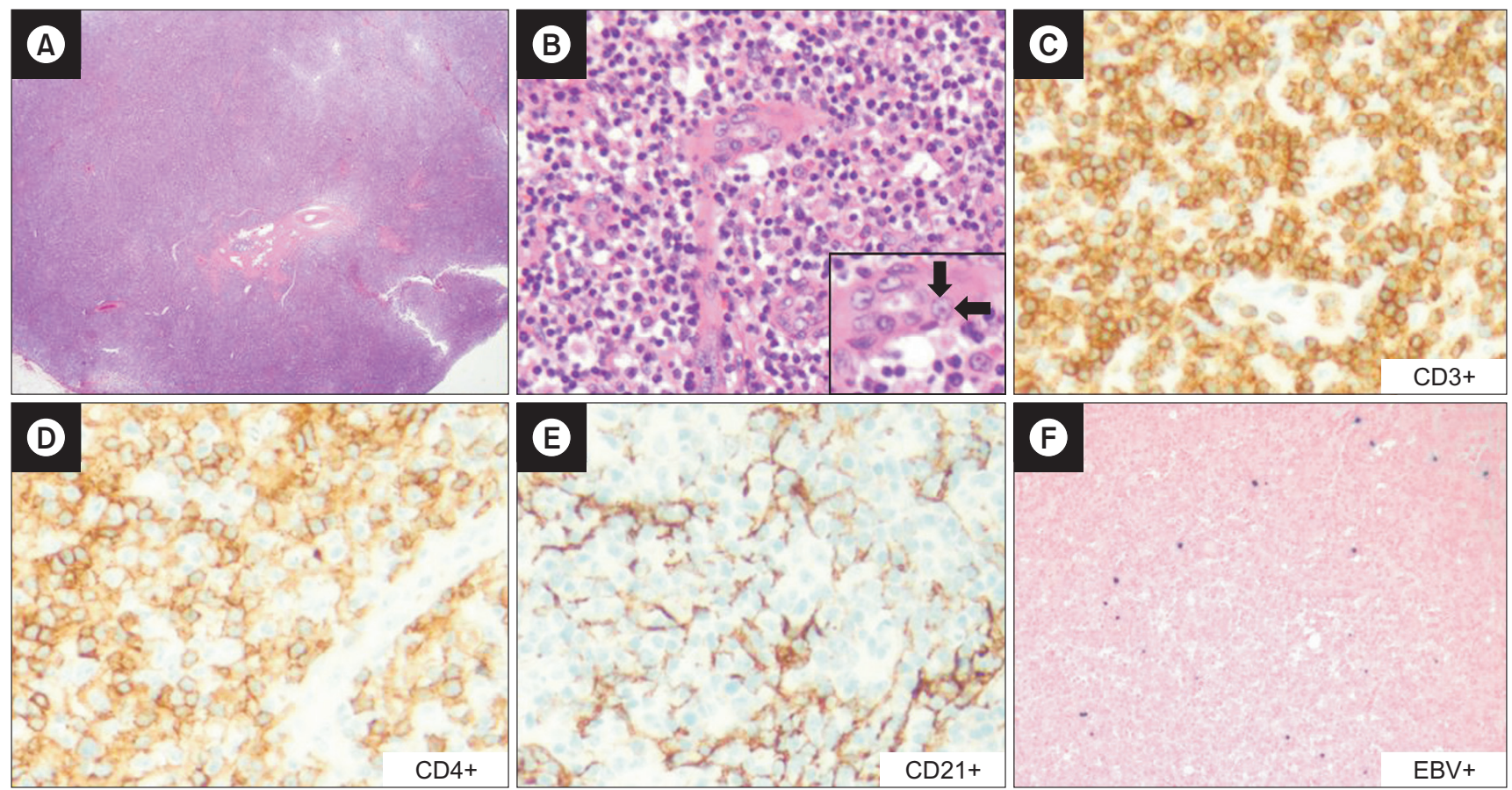

Fig. 3. Histopathologic Images from cervical lymph node dissection. (A) Hematoxylin-eosin (H\&E) staining showing effacement of nodal architecture with loss of germinal center $(H \& E, \times 12.5)$. (B) Small sized clear cells (detail in inset) (H\&E, $\times 200 ;$ inset, H\&E, $\times 400)$. (C, D) Immunohistochemical (IHC) features (T-cell marker; CD3, CD4 expression in neoplastic cells) (IHC, $\times 200)$. (E) Follicular dendritic cellular (FDC) marker CD21 expression in expanded extra follicular and perivascular FDC meshwork $($ IHC, $\times 200)$. (F) EpsteinBarr virus (EBV)-in situ hybridization: positive $(\mathrm{IHC}, \times 200)$. 
performed excision LN biopsy via selective neck dissection under general anesthesia. Histopathologic report of excision LN biopsy was angioimmunoblastic T-cell lymphoma (AITL) (Fig. 3A, 3B) with eligible immunohistochemical (IHC) staining and Epstein-Barr virus (EBV)-in situ hybridization results (Fig. $3 \mathrm{C}-3 \mathrm{~F}$ ). The patient was started on oral chemotherapy with cyclophosphamide (50 mg/day) due to her general condition (ECOG status 2-3). The patient is maintaining the cyclophosphamide (50 mg/day) without aggravation of previous symptoms.

AITL is a subtype of peripheral T-cell lymphoma (PTCL) in the proportion of $1-2 \%$ of all non-Hodgkin lymphomas (NHL) and 15-20\% of PTCLs [1]. AITL is a rare systemic disease clinically characterized by non-specific B-symptoms (fevers, unintentional weight loss, and/or drenching night sweats) with lymphadenopathy. Skin rash, hepatosplenomegaly and bone marrow infiltration are also seen in AITL patients. Evaluation of LN biopsy specimens is crucial diagnostic tool for the diagnosis of disease. Partial or total effacement of the LN architecture with small to medium atypical $\mathrm{T}$ cells with clear to pale cytoplasm is one of the favored histopathologic morphology of AITL. Plus, in immunophenotype, atypical cells are typically positive for CD3, CD4, and CD10, as well as markers of T-follicular helper (TFH) cells such as CD10, CXCL13, BCL6, and PD1. The atypical cells cluster close to high endothelial venules surrounded by follicular dendritic cells (FDCs) meshworks. CD21 may be performed that highlight the FDCs [2]. EBV infection and B cell-specific mutations may be important in AITL development. However, the correlation of the mutations and EBV infection is still unknown [2].

Several gene mutations associated with AITL were reported by gene expression profiling such as Tet Methylcytosine Dioxygenase 2 (TET2, 80\%), Isocitrate dehydrogenase (IDH2, 20\% to 30\%) and DNA methyltransferase $3 \mathrm{~A}$ (DNMT3A, 20\% to 30\%) [3]. Interestingly, the mutations in IDH isoforms (IDH1 and IDH2) have been also reported in cholangiocarcinoma [4]. IDH is an essential metabolic enzyme for cellular respiration in the tricarboxylic acid (TCA) cycle. This IDH isoforms mutant leads to aberrant cellular proliferation. However, because AITL is the rare and ag- gressive disease, there was no documented reports relation between AITL and cholangiocarcinoma until now.

Standard treatment for AITL have not been established yet. Chemotherapies for AITL most frequently used are cyclophosphamide, doxorubicin hydrochloride (hydroxydaunorubicin), vincristine sulfate (Oncovin), and prednisone (CHOP), CHOP with etoposide (ECHOP), cyclophosphamide, doxorubicin, vincristine, dexamethasone (hyperCAVD), or mitoxantrone, isophosphamide, mesna after each isophosphamide, etoposide (MINE) as the initial regimens [5]. However, it is difficult to use systemic chemotherapy for elderly with poor general condition. Furthermore, AITL is often relapses and refractory to chemotherapy. Therefore, novel treatment strategies for AITL are being studied.

\section{CONFLICTS OF INTEREST}

No potential conflict of interest relevant to this article was reported.

\section{AUTHOR'S CONTRIBUTIONS}

All authors discussed each other and conceived the idea to progress this study. Additionally, Seungjung Ha and Jae Keun Park directed the work and mainly drafted the article. Min Eui Hong performed the histopathological analysis with surgical specimens. All authors reviewed the manuscript.

\section{ORCID}

Seungjung Ha, https://orcid.org/0000-0001-6438-614X

Min Eui Hong, https://orcid.org/0000-0002-4409-4286

Jae Keun Park, https://orcid.org/0000-0002-3367-1770

\section{REFERENCES}

1. Swerdlow SH, Campo E, Pileri SA, et al. The 2016 revision of the World Health Organization classification of lymphoid neoplasms. Blood 2016;127:2375-2390.

2. Lunning MA, Vose JM. Angioimmunoblastic Tcell lymphoma: the many-faced lymphoma. Blood 
2017;129:1095-1102.

3. Lemonnier F, Gaulard P, de Leval L. New insights in the pathogenesis of T-cell lymphomas. Curr Opin Oncol 2018;30:277-284.

4. Grassian AR, Pagliarini R, Chiang DY. Mutations of isocitrate dehydrogenase 1 and 2 in intrahepatic cholangio- carcinoma. Curr Opin Gastroenterol 2014;30:295-302.

5. Tokunaga T, Shimada K, Yamamoto K, et al. Retrospective analysis of prognostic factors for angioimmunoblastic T-cell lymphoma: a multicenter cooperative study in Japan. Blood 2012;119:2837-2843. 